\title{
UNA MIRADA A LA APLICACIÓN DE LOS PRINCIPIOS DE CONTABILIDAD GENERALMENTE ACEPTADOS Y A LAS NORMAS INTERNACIONALES DE INFORMACIÓN FINANCIERA EN LAS PYMES DE CÚCUTA Y SU ÁREA METROPOLITANA.
}

\section{A LOOK AT THE APPLICATION OF GENERALLY ACCEPTED ACCOUNTING PRINCIPLES AND INTERNATIONAL FINANCIAL REPORTING STANDARDS IN SMES CUCUTA AND METROPOLITAN AREA.}

Nubia Isabel Díaz-Ortega ${ }^{1}$

Forma de citar: DÍAZ-ORTEGA Nubia Isabel. Una mirada a la aplicación de los principios de contabilidad generalmente aceptados y a las normas internacionales de información financiera en las PYMES de Cúcuta y su área metropolitana. Respuestas. 2014; 19(1):66-78.

Recibido:

Noviembre 4 de 2013

Aceptado:

Enero 16 de 2014

\section{RESUMEN}

Antecedentes: Las Normas Internacionales de Información Financiera (NIIF) son un conjunto de estándares y guías de calidad que se usan para preparar y reportar la información contable de la empresa, sin embargo, los principios de contabilidad generalmente aceptados rigen las prácticas contables actuales en la empresa. La aplicación de las (NIIF) permite proyectar un proceso contable sujeto a los posibles cambios que se presentarían en el proceso contable en general. Objetivo: El objetivo del presente estudio fue caracterizar el estado actual de los principios generales de la contabilidad y las (NIIF) en la mediana empresa de Cúcuta y su Área Metropolitana antes de la entrada en vigencia de las (NIIF). Métodos: Se utilizó el análisis de carácter documental y descriptivo fundamentado en los postulados teóricos de Bustamante (2013), Flórez (2013), Fuentes (2013), entre otros. Acompañado de un trabajo de campo con un diseño no experimental, transeccional, conformado por una muestra de 64 medianas empresas. Se utilizó la encuesta como instrumento de recolección de datos construida con 30 ítems con cinco posibilidades de respuesta, instrumento validado por juicio de expertos. Resultados: Las medianas empresas aplican los principios de contabilidad de una manera alta y la implementación de las (NIIF) es muy baja tanto en su cronograma, principios, tecnología, capacitación, entre otros. Conclusiones: Las medianas empresas se encuentran rezagadas en el nuevo proceso de implementación de las (NIIF) que presentarán cambios en los estados financieros por la aplicación de nuevos principios de valoración, medición y presentación de la información financiera.

Palabras clave: Principios de Contabilidad Generalmente Aceptados, Normas Internacionales de Información Financiera, Medianas Empresas. 


\section{ABSTRACT}

Background: The International Financial Reporting Standards (IFRS) are a set of quality standards and guidelines that are used to prepare and report accounting information from the company, however, generally accepted accounting principles governing the current accounting practices in company. The application of (IFRS) accounting allows forecasting process subject to possible changes that would arise in the accounting process in general. Objective: The objective of this study was to characterize the current state of the general principles of accounting and (IFRS) in the medium company of Cucuta and its metropolitan area before the effective date of (IFRS). Methods: The analysis of documentary and descriptive based on the theoretical postulates of Bustamante (2013), Flórez (2013), Fuentes (2013), among others was used. Accompanied by a field with a non-experimental, transactional design, consisting of a sample of 64 medium-sized companies. The survey instrument used for data collection constructed with 30 items with five possible answers, instrument validated by expert judgment. Results: Midsize companies apply accounting principles in a high fashion and the implementation of (IFRS) is very low both in its schedule, principles, technology, training, among others. Conclusions: Midsize companies are lagging behind in the new implementation process of (IFRS) changes presented in the financial statements by the application of new principles of assessment, measurement and presentation of financial information.

Keywords: Generally Accepted Accounting Principles, International Financial Reporting Standards, Medium Enterprises.

\section{INTRODUCCIÓN}

E propósito fundamental de la presente investigación es analizar los principios de contabilidad generalmente aceptados aplicados en las medianas empresas y el uso de las NIIF antes de su entrada en vigencia. Esta es una de las principales novedades para el contador de la época actual, cabe decir, en estos momentos de globalización económica se deben presentar los estados financieros con las mismas bases de elaboración para ser interpretados por sus usuarios desde cualquier parte del planeta, para convertir a los estados financieros en una herramienta valida, segura y confiable para que las empresas puedan tomar las decisiones financieras mas oportunas.
En consecuencia, el concepto de NIIF con el paso de tiempo se ha convertido en una obligación para las empresas colombianas, las cuales se encuentran en un regazo en este tema en el continente. Sin embargo, a través de normas legales se han realizado avances en el tema, como la división de las empresas por grupos, el establecimiento de un periodo de transición y preparación para elaborar el balance inicial antes de las normas, y la posterior aplicación plena. Las medianas empresa se encuentran clasificadas en este ranking, con un tiempo de advertencia para implementar este proceso.

Uno de los mayores retos para las medianas empresas en el campo de las NIIF, lo constituye 

internacionales de información financiera en las pymes de Cúcuta y su área metropolitana.

No. 1

Enero - Junio 2014

ISSN 0122-820X

PP: $66-78$ el hecho de lograr prepararse a tiempo para su aplicación. Lo que implica el análisis de los prácticas contables actuales, su situación en cuanto a la capacitación del recurso humano, de los sistemas tecnológicos, de la destinación de recursos económicos, para lo cual la normatividad establece hasta el 2016 para cumplir este proceso.

Lapresenteinvestigacióntrataráesencialmente de los principios generales de contabilidad y de las NIIF, aportando una breve descripción teórica de las principales exponentes de las variables del estudio. Además, muestra los resultados de la investigación de campo realizada que resalta la importancia del tema en los momentos actuales para la mediana empresa de Cúcuta y su Área Metropolitana.

\section{La situación de la mediana empresa de Cúcuta y su Área Metropolitana}

Cúcuta, capital de Norte de Santander es reconocida como la ciudad comercial e industrial que posee una de las zonas francas más activas del país y de América Latina. Por su ubicación en la zona fronteriza con Venezuela es famosa por tener una activa y reconocida industria láctea, de construcción, textiles, calzado y marroquinería.

A finales de 2011, según datos de la Cámara de Comercio de Cúcuta las exportaciones fueron de $\$ 406,2$ millones de dólares, en el periodo de enero a noviembre del año 2012 el monto fue de 378,6 millones de dólares, presentando una reducción de $\$ 27.6$ millones de dólares, influenciado por la crisis actual de la ciudad de Cúcuta y en general de Norte de Santander. Los empresarios aseguraron acerca de la situación económica actual de la ciudad así como de los municipios fronterizos con Venezuela que deberían tener un régimen especial y tributario al amparo de una emergencia económica por la postración que golpea la región.

SegúnMora (2013), presidentedelaFederación
Nacional de Comerciantes Seccional Cúcuta en entrevista para Confidencial Colombia, aseguró que la situación es tan delicada que al cierre del primer mes del año 2013, hay empresarios y comerciantes que reportan caídas en sus despachos por el orden del 80\%. La caída del comercio en 2012 sumó $40 \%$, básicamente por la contracción del último trimestre. $Y$ es de tener en cuenta que el bolívar fuerte que valía 1,80 pasó a 80 , es decir que la moneda venezolana que costaba a inicios de 2012, 220 pesos, a inicios del 2013 se consigue en 80 pesos.

Para Fenalco Cúcuta, puede darse la posibilidad que el gobierno de Venezuela disminuya en un $29 \%$ las importaciones de bienes, escenario apremiante al implicar que los dólares de esas operaciones de comercio exterior generará un déficit en divisas. Esta situación obligará a muchos agentes del mercado a conseguir dólares en el mercado negro disparando su valor, por cuanto Venezuela no es autosuficiente en materia de producción, acelerando con mayor vehemencia la devaluación en zona de frontera, estas empresas en crisis económica y comercial deben enfrentarse a la obligación de adaptar sus sistemas contables a las NIIF.

No obstante, esas mismas empresas en crisis económica y comercial deben enfrentarse al reto y a la obligación de adaptar sus sistemas contables a las NIIF; ciertamente, hoy en día la aplicación de la NIIF es vital para la mediana empresa de Cúcuta y su Área Metropolitana. Es el momento oportuno para que las empresas inicien el proceso de adopción de las NIIF, para que esta estandarización contable le permita integrarse al mercado financiero internacional, incrementar sus exportaciones y volverse más competitivas.

Ante esta situación obligatoria y obligante se hace necesario conocer los posibles beneficios que la adopción de las prenombradas NIIF signifique, así como, los costos inherentes a 
su adopción y la adaptación de los sistemas contables actuales a las mismas. Por supuesto que el conocer los parámetros anteriores relativos a los beneficios esperados y a los costos necesarios para la adopción de las NIIF, origina la necesidad de investigar, en la comunidad empresarial de la ciudad de Cúcuta y su Área metropolitana, los efectos previsibles de la implementación y puesta en práctica de esas novedosas normas internacionales.

Principalmente las medianas empresas del Norte de Santander, en general y las de Cúcuta y su Área Metropolitana en particular, tienen relaciones internacionales con el mundo, a través de sus exportaciones e importaciones no sólo de bienes, sino también de servicios y de capitales, lo que obliga no solamente al cumplimiento legal de las NIIF, sino también a su implantación. En el mundo globalizado actual las empresas requieren de alianzas estratégicas de todo tipo, entre las cuales la conformación de empresas de capital internacional es una posibilidad: lo que podría convertirse en una ventaja competitiva para aquellas empresas que adapten con todo rigor las NIIF, ya que podría comunicarse con sus aliados estratégicos internacionales de una manera más fluida.

\section{Principios de contabilidad generalmente aceptados en Colombia}

En estos momentos, en Colombia el decreto 2649 de 1993 reglamenta la contabilidad en general y expide los principios o normas de contabilidad generalmente aceptados en Colombia denominados PCGA. Se definen como el conjunto de conceptos básicos y reglas que deben ser observados al registrar e informar contablemente sobre los asuntos y actividades de los entes económicos.

El primer principio es el de ente económico. Según Bustamante (2013) los estados financieros se refieren siempre a un ente donde el elemento subjetivo o propietario es considerado como tercero. Este supuesto define que todo estado financiero debe hacer referencia a una entidad en la cual los propietarios o accionistas se consideran como terceros, por tal motivo se deben separar el patrimonio personal del propietario o del dueño, del patrimonio de la entidad.

El segundo principio es el de continuidad también denominado empresa en marcha. Según Gavelán (2013) se basa en la presunción de que la empresa continuará sus operaciones por un tiempo indefinido y no será liquidado en un futuro previsible, salvo que existan situaciones como: significativas y continuas pérdidas, insolvencia, etc. En consecuencia, la valuación contable de los hechos serán los mismos u homogéneos, mientras no cambie su situación o cese en sus actividades, en cuyo caso se aplicará criterios de realización o de mercado.

El tercer principio es el de unidad de medida. Según Bustamante (2013) generalmente se utiliza como moneda de cuenta el dinero que tiene curso legal en el país dentro del cual funciona el "ente" y en este caso el " precio" esta dado en unidades de dinero de curso legal.

El cuarto principio es el de período contable. Establece el decreto 2649/93 que los estados financieros deben tener un corte a 31 de diciembre para la presentación e sus estados financieros de propósito general, se toma como periodo contable un espacio de tiempo de una año.

El quinto principio es el de medición. Según Bustamante (2013) los resultados económicos solo deben computarse cuando sean realizados, o sea cuando la operación que los origina queda perfeccionada desde el punto de vista de la legislación o prácticas comerciales aplicables y se hayan ponderado fundamentalmente todos los riesgos inherentes a tal operación. Debe establecerse con carácter general que el concepto "realizado" participa del concepto devengado.
Enero - Junio 2014

ISSN 0122-820X

PP: 66 - 78 
No. 1

Enero - Junio 2014 ISSN 0122-820X

PP: $66-78$

70
El sexto principio es el de esencia sobre la forma. El Decreto 2649/93 indica que los recursos y hechos económicos deben ser reconocidos y revelados de acuerdo con su esencia o realidad económica y no únicamente en su forma legal. Es decir, prevalecen los hechos sobre la formalidad.

El séptimo principio es el de realización. Según Bustamante (2013), los resultados económicos solo deben computarse cuando sean realizados, o sea cuando la operación que los origina queda perfeccionada desde el punto de vista de la legislación o prácticas comerciales aplicables y se hayan ponderado fundamentalmente todos los riesgos inherentes a tal operación.

El octavo principio es el de asociación de ingresos y gastos. El decreto 2649/93 señala que se deben asociar con los ingresos devengados en cada período los costos y gastos incurridos para producir tales ingresos, registrando unos y otros simultáneamente en las cuentas de resultados.

El noveno principio es el de mantenimiento del patrimonio. El decreto 2649/93 considera que un ente económico obtiene utilidad o excedentes, en un período únicamente después de que su patrimonio al inicio del mismo, excluidas las transferencias de recursos a otros entes realizadas conforme a la ley, haya sido mantenido o recuperado. Esta evaluación puede hacerse respecto del patrimonio financiero (aportado) o del patrimonio físico (operativo).

El décimo principio es el de revelación. En análisis de Bustamante (2013), con base en el principio de revelación suficiente, se ha pensado en la necesidad de ajustar los estados financieros con motivo de los cambios en el poder adquisitivo de la moneda. El contador público está obligado a proporcionar información fidedigna que permita al usuario de tal información tomar decisiones acertadas. Ha sido una buena costumbre entre contadores el incluir notas aclaratorias que puedan tener una empresa y demás información que pueda ser útil para el lector de dichos estados financieros.

Finalmente, el decreto 2643/93 cierra el listado con los principios de materialidad y prudencia. El primero, según Gavelan (2013), el principio de significación, también denominado materialidad, está dirigido complementariamente a dos aspectos principales de la contabilidad: cuantificación o medición del patrimonio y exposición de partidas en los estados financieros. El segundo según Bustamante (2013) significa que cuando se deba elegir entre dos valores por un elemento del activo, normalmente se debe optar por el más bajo, o bien que una operación se contabilice de tal modo que la alícuota del propietario sea menor. Este principio general se puede expresar también diciendo: "contabilizar todas las pérdidas cuando se conocen y las ganancias solamente cuando se hayan realizado".

\section{Perspectivas de las Normas Internacionales de Información Financiera}

Daniels, Radebaugh y Sullivan (2004), hacen referencia que las NIIF se crean con el fin de desarrollar normas que contaran con una aceptación rápida y amplia enfocándose a mejorar la revelación de la información. Estas normas permitirían a las empresas extranjeras cotizar en sus bolsas de valores sin la necesidad de tener que conciliar sus estados financieros con los diferentes PCGA de cada país y a pensar de las grandes diferencias de normas que hay en el mundo.

Por otra parte, según los autores anteriormente citados las NIIF conducen hacia la armonización como el camino para una tendencia hacia proporcionar información compatible con las necesidades de los inversionistas a la integración global de los mercados de capital. Esto significa que los inversionistas tienen un acceso más fácil 
y rápido a las oportunidades de inversión alrededor del mundo.

Por lo tanto, se necesita información financiera equiparable por la necesidad de las EMN (empresas multinacionales) de obtener capital fuera de los mercados de capital de su país de origen, genera al mismo tiempo el menor número posible de estados financieros. La armonización regional, política y económica, como los esfuerzos de la UE (Unión Europea) afectan la contabilidad. Los aspectos comerciales, de inversión y las presiones de las EMN hacia normas más uniformes que faciliten la elaboración de informes generales de cada país y reduzcan sus costos.

Estas circunstancias, colaboran con poder dar respuesta a los seis aspectos principales que difieren los estados financieros de un país con los de los otro país como lo son: idioma, moneda, tipo de estado, formato del estado financiero, grado de las revelaciones en las notas al pie y por último los PCGA en los que se basan los estados financieros de cada país. Las NIIF buscan finalmente la armonización de estos aspectos.

En igual forma, Doupnik y Perera (2008) describen las NIIF, como el cuerpo de pronunciamientos autoritativos emitidos $\mathrm{O}$ adoptados por el Consejo Internacional de Normas Internacionales. Las NIIF se han adoptado como los PCGA para las compañías inscritas en bolsas de valores para propósitos de inscripciones cruzadas, ellos mencionan que hay varias diferencias entre las NIIF y los principios de PCGA, y hacen referencia a las diferencias en definición, reconocimiento, medición, alternativas que ofrecen, también en la falta de requisitos o de lineamientos, la diferencia de presentación y por ultimo diferencias en la revelación. Ellos concluyen que en muchos casos las NIIF son más flexibles que los PCGA, además que las NIIF tienen menos lineamientos altamente ilustrativos que los PCGA de Estados Unidos.
También Flórez (2013) especialista de las NIIF habla su marco conceptual definiendolo como los fundamentos de la información que deben contener los estados financieros, procurando como objetivo y cualidad de la información tengan unos elementos para cada estado financiero. Menciona que al registrar una transacción se debe tener en cuenta cual es la finalidad de las NIIF. El reconocimiento es una nueva técnica de los estándares internacionales es la incorporación de una partida al estado, y su situación financiera el resultado cuando el ingreso o salida de dicha partida ocasione beneficios económicos para que la partida pueda ser valorada fiablemente.

Concluye que va muy ligado al registro contable, a la causación o devengo donde se va a tener en cuenta el momento de hacer el reconocimiento, el análisis individualmente de cada partida porque cada una de ellas tiene una norma, una regla o un estándar y es por ella que se va a aplicar su manejo. Agrega, el anterior autor dentro de sus múltiples publicaciones la definición de comparabilidad como un principio que busca evaluar diferencias, determinar en qué contexto se dio, esta comparabilidad permite evaluar tendencias y comparar políticas distintas.

Asimismo, Cárdenas (2012) en su artículo "NIIF mas que un requisito", sostiene la existencia un conjunto único y global de normas de contabilidad y de información financiera se hace cada vez más real, las empresas colombianas deben ver en las NIIF una oportunidad para fortalecerse ganando espacio en el mercado globalizado. Más que una obligación legal que debe cumplirse, las NIIF se convierten en una herramienta de posicionamiento de las empresas en el exterior. SN 0122-820X P: $66-78$

Fuentes (2013) define a las NIIF como normas contables mundiales de elevada calidad, comprensibilidad y de cumplimiento obligatorio que llevan a requerir información comparable, transparencia y de elevada calidad 
No. 1

Enero - Junio 2014 ISSN 0122-820X

PP: $66-78$

en los estados financieros para propósitos gerenciales. Las NIIF son normas contables con sus respectivas interpretaciones y emitidas por el Comité de Normas Internacionales de Contabilidad (IASB).

Por otra parte, el Consejo Mexicano de Normas de Información Financiera, A.C. indica que las normas deben ser de alta calidad y deben dar como resultado información comparable y transparente a escala internacional y proporcionar una revelación completa. Los inversionistas deben ser capaces de realizar un análisis significativo del desempeño de la empresa o entidad económica a través de períodos de tiempo, además de entre compañías.

Atendiendo a lo dicho, si el fin de las normas contables es satisfacer el objetivo de registrar e informar operaciones y eventos, en cualquier lugar y tiempo en que se realicen, los auditores y organismos reguladores insistirán en obtener una rigurosa interpretación y aplicación de las mismas, de lo contrario, la comparabilidad y la transparencia que son la finalidad de los estándares comunes no se logrará. Las normas contables de alta calidad son vitales para las funciones de regulación del mercado de capital que ejercen los organismos reguladores nacionales e internacionales.

\section{Situación de las NIIF en Colombia}

En Colombia, el proceso de convergencia a NIIF inicio con la ley 1314 de 2009 que le dio atribuciones al Consejo Técnico de la Contaduría como organismo rector de este proceso. A partir de este momento, a través de un debido proceso se crearon tres grupos de empresas para la aplicación de la NIIF plenas, pymes y contabilidad simplificada.

El primer grupo, acoge las más grandes empresas emisoras de valores, con interés público y entidades grandes con requisitos especiales. En el segundo grupo, clasifican las empresas de tamaño grande y mediano, que no sean emisoras de valores ni tengan interés público, ni cumplan requisitos especiales del primer grupo. El tercer grupo, se compone de las microempresas según la ley colombiana que no pertenezcan al grupo 2 .

Asimismo, en Colombia las empresas se clasifican de acuerdo la ley 905 de 2004, la cual define las medianas empresas como toda unidad de explotación económica, realizada por persona natural o jurídica, en actividades empresariales, agropecuarias, industriales, comerciales o de servicio, rural o urbano. Deben responder a dos (2) de los siguientes parámetros: Planta de personal entre cincuenta y uno (51) y doscientos (200) trabajadores, ó activos totales por valor entre cinco mil uno (5.001) a treinta mil (30.000) salarios mínimos mensuales legales vigentes.

Es decir, estas medianas empresas clasificarían según las NIIF en el grupo dos NIIF para PYMES, por tanto deben aplicar el Decreto 3022 de 2013, que en general omiten temas no relevantes para PYMES, algunos tratamientos contables de las NIIF plenas están prohibidos y tiene simplificaciones, las revelaciones son reducidas. En otras palabras, tienen un menor contenido que las NIIF plenas otorgándoles un menor relación beneficio costo a los empresarios en el momento de iniciar la implementación de las NIIF.

Igualmente, el cronograma de aplicación de las NIIF para el grupo dos inicia con un período obligatorio de preparación que es el año 2014, una fecha de transición y su balance de apertura a NIIF finalizando el año 2015. Por lo tanto, con la aplicación de los primeros estados financieros no comparativos con NIIF para PYMES al 31 de diciembre de 2016 convirtiéndose en el primer periodo contable de aplicación de NIIF.

\section{Tipo y Diseño de investigación}

Este estudio según las características de las variables principios de contabilidad 
generalmente aceptados y las NIIF, se puede catalogar dentro del enfoque cuantitativo. De acuerdo a Lerma (2009), la investigación cuantitativa se caracteriza por plantear hipótesis que pueden presentarse como proposiciones, que pueden fácilmente ser convertidas en formulas matemáticas. Además se utilizan técnicas estadísticas estructuradas para el análisis de la información.

De acuerdo a Bernal (2010), la definición del diseño de investigación está determinada por el tipo de investigación que va a realizarse y por la hipótesis que va a aprobarse durante el desarrollo de la investigación. En atención al diseño esta investigación se clasifica en: no experimental, transeccional- descriptivo, de campo, los cuales se describen a continuación.

En virtud a la población en estudio, a efectos de la presente investigación se consideraron las empresas constituidas legalmente como medianas empresas según el registro de la Cámara de Comercio de Cúcuta. De tal manera, la población quedó conformada por un universo de 174 empresas de las cuales, al aplicar la fórmula del muestreo estadístico simple con un nivel de confiabilidad del $95 \%$ y un margen de error del $5 \%$, se obtuvo una muestra de 64 medianas empresas. Las empresas fueron seleccionadas al azar ya que todas las empresas cumplían con las características de la población y tenían la misma posibilidad de ser elegidas. Se les aplicó una encuesta como instrumento de recolección de información, conformada por 30 ítems con una escala de Lickert de cinco opciones de respuesta. (Ver Apéndice No. 1 - Encuesta).

\section{RESULTADOS DE LA INVESTIGACIÓN}

Sobre la anterior base, al analizar los resultados de la variable principios de contabilidad generalmente aceptados se obtiene una alta valoración por parte de los contadores de las medianas empresas con una media del 4.29. Es importante referenciar que los resultados fueron altos para varios principios de contabilidad como período, unidad de medida, entre otros. No obstante, algunas áreas son susceptibles de mejoras debiendo ser intervenidas con orientaciones específicas, como por ejemplos los principios de actividad y periodo que son considerados moderadamente por estas empresas con medias del 3.20 y 2.86 respectivamente.

Tabla 1. Variable Principios de Contabilidad Generalmente Aceptados

\begin{tabular}{|c|c|c|c|c|c|}
\hline \multicolumn{6}{|c|}{ Variable Principios de Contabilidad Generalmente Aceptados } \\
\hline NUNCA & $\begin{array}{c}\text { CASI } \\
\text { NUNCA }\end{array}$ & AVECES & $\begin{array}{c}\text { CASI } \\
\text { SIEMPRE }\end{array}$ & SIEMPRE & TOTAL \\
\hline $1-2 \%$ & $3-5 \%$ & $8-12 \%$ & $20-31 \%$ & $32-50 \%$ & 64 \\
\hline
\end{tabular}

Esta tabla 1 presenta en términos generales el promedio de las respuestas dadas por los contadores de las medianas empresas de Cúcuta y su Área Metropolitana a cada uno de los ítems. Se infiere que el 50\% de las empresas siempre aplica los principios de contabilidad generalmente aceptados, $31 \%$ lo hace casi siempre, el $12 \%$ a veces, mientras que el $5 \%$ casi nunca y el otro $2 \%$ restante nunca. Es evidente que las empresas tienen los principios de contabilidad generalmente aceptados al momento de registrar las operaciones económicas.

Ahora bien, hay coincidencias al confrontar los resultados con la normatividad existente en Colombia que implican que las empresas analizadas se orientan a aplicar por obligación legal los principios de contabilidad generalmente aceptados como el conjunto de preceptos que deben ser tenidos en cuenta al momento del registro de las operaciones económicas.

Enero - Junio 2014

ISSN 0122-820X

PP: $66-78$

Por otra parte, a nivel de la variable NIIF, se obtuvo que es bajo el nivel de aplicación 
No. 1

Enero - Junio 2014

ISSN 0122-820X

PP: $66-78$

74 de las mismas por parte de las medianas empresas los resultados arrojaron una media del 1.38 demostrando baja competencia para comprender los nuevos criterios de medición, las características y revelación de la información contable.

Tabla 2. Variable Normas Internacionales de Información Financiera

\begin{tabular}{|c|c|c|c|c|c|}
\hline \multicolumn{5}{|c|}{ Variable 2. Normas internacionales de información financiera } \\
\hline NUNCA & $\begin{array}{c}\text { CASI } \\
\text { NUNCA }\end{array}$ & AVECES & $\begin{array}{c}\text { CASI } \\
\text { SIEMPRE }\end{array}$ & SIEMPRE & TOTAL \\
\hline $44-56 \%$ & $16-25 \%$ & $4-6 \%$ & & & 64 \\
\hline
\end{tabular}

En la tabla 2 se puede apreciar en términos generales el promedio de las respuestas dadas por los contadores de las medianas empresas de Cúcuta y su Área Metropolitana a cada uno de los ítems sobre NIIF. Los resultados arrojan que $69 \%$ de las empresas nunca aplica las NIIF, el $25 \%$ casi nunca y el $6 \%$ a veces, demostrándose una baja utilización de las NIIF.

En síntesis, que la importancia de las NIIF radican en que tienen unos elementos para cada estado financiero, debiendo hacerse un análisis individual de cada partida porque cada una de ellas tiene una norma, una regla o un estándar que establece su manejo. El análisis de los resultados evidencia que la variable normas internacionales no es valorada por los contadores de las medianas empresas. Dicha valoración global se considera una debilidad pues representa una falta de conocimiento de un tema tan vital para la comunidad contable en los momentos actuales.

\section{CONCLUSIONES}

En relación al primer objetivo específico, se caracterizaron los principios de contabilidad generalmente aceptados aplicados por las medianas empresas de Cúcuta, encontrándose que fue alto su nivel indicando que contribuyen con el buen desarrollo de los sistemas de contabilidad actuales de las empresas. Así como moderado respecto al principio de actividad y periodo no identificándose plenamente con los resultados de los principios de contabilidad generalmente aceptados.

Respecto al segundo objetivo específico, se identificaron las NIIF, indicándose que se utilizan en nivel bajo sus políticas contables. Es bajo el conocimiento en el registro de los hechos económicos bajo NIIF, aplicación de sus criterios de valoración, revelación de los estados financieros y en un bajo presupuesto para iniciar la transición. Lo antes mencionado dio como balance un nivel bajo, siendo desfavorable para la armonización de la información contable de las medianas empresas de Cúcuta.

Se hace necesario implementar acciones concretas de capacitación en las que participen unidas las universidades con facultades de contaduría en la región y las medianas empresas. El fin es fortalecer los planes de trabajo respecto a NIIF, que incluyan los aspectos como el diagnóstico, la sensibilización y el cambio tecnológico propio de este proceso.

\section{BIBLIOGRAFÍA}

Bernal, C. (2010). Metodología de la investigación. Administración, economía, humanidades y ciencias sociales. Editorial Pearson. Bogotá.

Bustamante, E. (2013). Los principios de contabilidad generalmente aceptados. Consultado el 2 de mayo de 2013. Disponible en : http://es.scribd.com/doc/3240247/ Principios-Contables-GeneralmenteAceptados CAMARA DE COMERCIO DE CUCUTA, (2010). Página web en línea. Disponible en: htpp://www.cccucuta.org.co

Cárdenas Lesmes, Rosa María. (2012). NIIF, más que un requisito. Revista Portafolio. Bogotá. 
Consejo Mexicano de Normas de Información

Financiera. (2013). Página web en línea.

Disponible en: htpp://www.cinif.org.mx/.

Daniels, Radebaugh y Sullivan. (2004). Negocios internacionales. Ambientes y operaciones. México. Pearson Prentice Hall.

Decreto 2643 (1993). Diario Oficial de Colombia, 41.156. Diciembre 29, 1993.

Doupnik, T. y Perera H. (2008). Contabilidad internacional. Editorial Mc Graw Hill. México

Fenalco Norte de Santander. (2013). Página web en línea. Disponible en: htpp:/www. fenalconortedesantander.com/ Flórez, E. (2013). Las NIIF en Colombia. Consultado el 18 de mayo de 2013. Disponible en :http:// www.youtube.com/watch?v=_nqS_YzdxTQ

Fuentes. J. (2013). Seminario de titulación en contabilidad de su proyecto finanzas internacionales y postulados de contabilidad internacional. Centro de Estudios IETE. México.

Gavelán, J. (2013). Los principios de contabilidad. Consultado el 5 de mayo del 2013. Disponible en: http://sisbib. unmsm.edu.pe/bibvirtual/publicaciones/ quipukamayoc/2000/primer/princi_conta.htm Lerma. H. (2009). Metodología de la investigación. Propuesta, anteproyecto y proyecto. Ecoe Ediciones. Bogotá.

Ley 1314 Convergencia Contable (2010). Diario Oficial de Colombia, 47.409. Julio 13, 2010.

Ley 590 Promoción a la mediana empresa (2000). Diario Oficial de Colombia, 41.434. Agosto 12, 2000.

Ley 905 Promoción a la mediana empresa (2004). Diario Oficial de Colombia, 45.628. Mayo 20, 2004. 
No. 1

\section{Apéndice No. 1- Encuesta}

PCGA- NIIFF EN MEDIANAS EMPRESAS

(Dirigido a los Contadores Públicos que laboran en Medianas Empresas - Cúcuta y Área Metropolitana)

\begin{tabular}{|c|c|c|c|c|c|c|}
\hline $\mathbf{N}^{\circ}$ & Ítems & I & $\mathrm{CN}$ & AV & $\mathrm{CS}$ & $\mathrm{S}$ \\
\hline 1 & La empresa emite los estados financieros por lo menos una vez al año & & & & & \\
\hline 2 & $\begin{array}{l}\text { La información financiera incluye el valor histórico, valor actual, valor presente y de } \\
\text { realización exclusiva relación con la empresa. }\end{array}$ & & & & & \\
\hline 3 & La contabilidad de la empresa, destaca la asociación de ingresos con su costo o gasto. & & & & & \\
\hline 4 & $\begin{array}{l}\text { Las operaciones mercantiles, son registradas en hechos económicos realizados con su } \\
\text { sentido financiero con su sentido financiero. }\end{array}$ & & & & & \\
\hline 5 & $\begin{array}{l}\text { Los datos contables que se presentan proporcionan información económica necesaria } \\
\text { para la toma decisiones. }\end{array}$ & & & & & \\
\hline 6 & Los hechos económicos no reconocidos se presentan en notas. & & & & & \\
\hline 7 & $\begin{array}{l}\text { La unidad monetaria (Pesos), es la base utilizada para medir las operaciones } \\
\text { mercantiles. }\end{array}$ & & & & & \\
\hline 8 & $\begin{array}{l}\text { La información financiera es presentada en la unidad monetaria establecida } \\
\text { (Pesos). }\end{array}$ & & & & & \\
\hline 9 & $\begin{array}{l}\text { Las partidas realizadas por la empresa que no se pueden asociar con un ingreso se } \\
\text { registran en siguiente periodo. }\end{array}$ & & & & & \\
\hline 10 & Los hechos económicos se registran de acuerdo con la realidad Económica. & & & & & \\
\hline 11 & $\begin{array}{l}\text { La contabilidad muestra las utilidades o excedentes después de haber mantenido o } \\
\text { recuperado el patrimonio. }\end{array}$ & & & & & \\
\hline 12 & La información contable, se revela de completa aunque resumida. & & & & & \\
\hline 13 & $\begin{array}{l}\text { La empresa hace el reconocimiento y presentación de los hechos económicos de } \\
\text { acuerdo a su importancia. }\end{array}$ & & & & & \\
\hline 14 & Las cifras de los estados financieros son presentadas en valores históricos. & & & & & \\
\hline 15 & $\begin{array}{l}\text { Las operaciones económicas se realizan teniendo en cuenta no sobreestimar activos e } \\
\text { ingresos no subestimar pasivos y gastos. }\end{array}$ & & & & & \\
\hline 16 & $\begin{array}{l}\text { Las transacciones económicas se registran, teniendo en cuenta las características y } \\
\text { prácticas de cada actividad. }\end{array}$ & & & & & \\
\hline 17 & La información contable que se presenta indica el período en el cual se efectuó. & & & & & \\
\hline 18 & $\begin{array}{l}\text { Las erogaciones de dinero son identificadas con el ingreso que originaron, } \\
\text { independientemente de su fecha de pago. }\end{array}$ & & & & & \\
\hline 19 & $\begin{array}{l}\text { Las partidas de los estados financieros poseen un valor que puede ser medido con } \\
\text { confiabilidad. }\end{array}$ & & & & & \\
\hline 20 & $\begin{array}{l}\text { Cuando se realiza una estimación sobre bases razonables se reconoce en la } \\
\text { contabilidad. }\end{array}$ & & & & & \\
\hline 21 & $\begin{array}{l}\text { Las partidas contables tienen importancia cuando un cambio modifica la decisión de } \\
\text { los interesados en ella. }\end{array}$ & & & & & \\
\hline 22 & $\begin{array}{l}\text { La empresa analiza las partidas que puedan tener un efecto importante en los estados } \\
\text { financieros. }\end{array}$ & & & & & \\
\hline 23 & Las políticas contables son empleadas uniformemente aplicando las mismas políticas. & & & & & \\
\hline
\end{tabular}




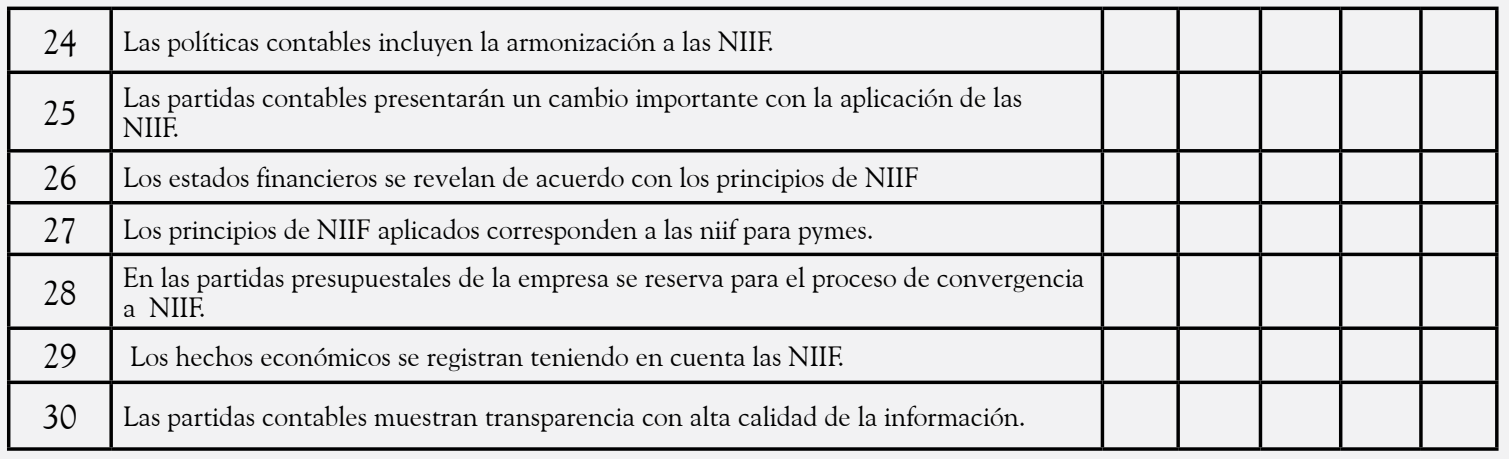


Respuestas

Cúcuta-Colombia

Vol. 19

No. 1

Enero - Junio 2014

ISSN 0122-820X

PP: $66-78$

78
Una mirada a la aplicación de los principios de contabilidad generalmente aceptados y a las normas internacionales de información financiera en las pymes de Cúcuta y su área metropolitana.

Apéndice No. 2- Resultados de la investigación

\begin{tabular}{|c|c|c|c|c|c|c|c|c|c|c|c|}
\hline PREGUNTA & SIEMPRE & & $\begin{array}{c}\text { CASI } \\
\text { SIEMPRE }\end{array}$ & & A VECES & & $\begin{array}{c}\text { CASI } \\
\text { NUNCA }\end{array}$ & & NUNCA & & MEDIA \\
\hline 1 & 62 & $97 \%$ & 2 & $3 \%$ & & $0 \%$ & & $0 \%$ & & $0 \%$ & 4.97 \\
\hline 2 & 44 & $69 \%$ & 12 & $19 \%$ & 2 & $3 \%$ & 5 & $8 \%$ & 1 & $2 \%$ & 4.4 \\
\hline 3 & 52 & $81 \%$ & 9 & $14 \%$ & 1 & $2 \%$ & 2 & $3 \%$ & & $0 \%$ & 4.73 \\
\hline 4 & 51 & $80 \%$ & 12 & $19 \%$ & 1 & $2 \%$ & & $0 \%$ & & $0 \%$ & 4.78 \\
\hline 5 & 56 & $88 \%$ & 7 & $11 \%$ & 1 & $2 \%$ & & $0 \%$ & & $0 \%$ & 4.85 \\
\hline 6 & 25 & $39 \%$ & 21 & $33 \%$ & 9 & $14 \%$ & 6 & $9 \%$ & 3 & $5 \%$ & 3.92 \\
\hline 7 & 50 & $78 \%$ & 13 & $20 \%$ & & $0 \%$ & & $0 \%$ & 1 & $2 \%$ & 4.73 \\
\hline 8 & 44 & $69 \%$ & 18 & $28 \%$ & & $0 \%$ & 1 & $2 \%$ & 1 & $2 \%$ & 4.61 \\
\hline 9 & 24 & $38 \%$ & 23 & $36 \%$ & 4 & $6 \%$ & 1 & $2 \%$ & 12 & $19 \%$ & 3.72 \\
\hline 10 & 47 & $73 \%$ & 13 & $20 \%$ & 3 & $5 \%$ & & $0 \%$ & 1 & $2 \%$ & 4.64 \\
\hline 11 & 42 & $66 \%$ & 17 & $27 \%$ & 3 & $5 \%$ & & $0 \%$ & 2 & $3 \%$ & 4.51 \\
\hline 12 & 42 & $66 \%$ & 14 & $22 \%$ & 2 & $3 \%$ & & $0 \%$ & 6 & $9 \%$ & 4.3 \\
\hline 13 & 42 & $66 \%$ & 14 & $22 \%$ & 6 & $9 \%$ & 1 & $2 \%$ & 1 & $2 \%$ & 4.5 \\
\hline 14 & 23 & $36 \%$ & 19 & $30 \%$ & 12 & $19 \%$ & 5 & $8 \%$ & 5 & $8 \%$ & 3.7 \\
\hline 15 & 32 & $50 \%$ & 20 & $31 \%$ & 8 & $13 \%$ & 3 & $5 \%$ & 1 & $2 \%$ & 4.2 \\
\hline 16 & 46 & $72 \%$ & 17 & $27 \%$ & 1 & $2 \%$ & & $0 \%$ & & $0 \%$ & 3.2 \\
\hline 17 & & $0 \%$ & & $0 \%$ & 54 & $84 \%$ & 9 & $14 \%$ & 1 & $2 \%$ & 2.86 \\
\hline 18 & 37 & $58 \%$ & 16 & $25 \%$ & 6 & $9 \%$ & 3 & $5 \%$ & 2 & $3 \%$ & 4.36 \\
\hline 19 & 46 & $72 \%$ & 6 & $9 \%$ & 2 & $3 \%$ & 9 & $14 \%$ & 1 & $2 \%$ & 4.04 \\
\hline 20 & 34 & $53 \%$ & 20 & $31 \%$ & 8 & $13 \%$ & 1 & $2 \%$ & 1 & $2 \%$ & 4.51 \\
\hline 21 & 39 & $61 \%$ & 20 & $31 \%$ & 4 & $6 \%$ & 1 & $2 \%$ & & $0 \%$ & 4.5 \\
\hline 22 & 49 & $77 \%$ & & $0 \%$ & 13 & $20 \%$ & 2 & $3 \%$ & & $0 \%$ & 4.4 \\
\hline 23 & 31 & $48 \%$ & 28 & $44 \%$ & 5 & $8 \%$ & & $0 \%$ & & $0 \%$ & 4.3 \\
\hline 24 & & $0 \%$ & & $0 \%$ & 2 & $3 \%$ & 4 & $6 \%$ & 58 & $91 \%$ & 1.1 \\
\hline 25 & & $0 \%$ & & $0 \%$ & 2 & $3 \%$ & 3 & $5 \%$ & 59 & $92 \%$ & 1.1 \\
\hline 26 & & $0 \%$ & & $0 \%$ & 2 & $3 \%$ & 3 & $5 \%$ & 59 & $92 \%$ & 1.04 \\
\hline 27 & & $0 \%$ & & $0 \%$ & 4 & $6 \%$ & 16 & $25 \%$ & 44 & $69 \%$ & 1.38 \\
\hline 28 & & $0 \%$ & & $0 \%$ & 16 & $25 \%$ & 14 & $22 \%$ & 34 & $53 \%$ & 1.72 \\
\hline 29 & & $0 \%$ & & $0 \%$ & 3 & $5 \%$ & 2 & $3 \%$ & 59 & $92 \%$ & 1.08 \\
\hline 30 & 12 & $19 \%$ & 20 & $31 \%$ & 3 & $5 \%$ & 10 & $16 \%$ & 19 & $30 \%$ & 2.93 \\
\hline
\end{tabular}

